\title{
TIDAL DISSIPATION IN STARS AND GIANT PLANETS: JEAN-PAUL ZAHN'S PIONEERING WORK AND LEGACY
}

\author{
S. Mathis ${ }^{1}$
}

\begin{abstract}
In this lecture opening the session focused on tides in stellar and planetary systems, I will review the Jean-Paul Zahn's key contributions to the theory of tidal dissipation in stars and fluid planetary layers. I will first recall the general principles of tidal friction in celestial bodies. Then, I will focus on the theories of the stellar equilibrium and dynamical tides founded by Jean-Paul and their predictions for the evolution of binary stars. I will underline their essential legacy for ongoing studies of tidal dissipation in stars hosting planets and in fluid planetary regions. I will also discuss his pioneering work on the turbulent friction applied on tidal flows by stellar convection and the corresponding still unsolved challenging problems. Next, I will present the results we obtained on tidal dissipation in the potential dense rocky/icy core of gaseous giant planets such as Jupiter and Saturn within the Encelade international team. This mechanism provides important keys to interpret the high-precision astrometric measurements of the rates of tidal orbital migration of the moons of these planets, which are found to be larger than expected. This corresponds to a Jovian and Saturnian tidal frictions which are higher by one order of magnitude than the usually used values calibrated on formation scenarios. Finally, I will review the work done by Jean-Paul and Michel Rieutord on potential Ekman boundary layers associated to tidal flows. As a consequence, a coherent physical modeling of tides is now mandatory to understand the properties and the evolution of stellar and planetary systems. To progress on this forefront research subject, we are walking on the path first drawn by Jean-Paul.
\end{abstract}

\section{Introduction}

In this contribution, I will try to provide the more global and synthetic overview as possible of the key contributions of Jean-Paul to the study of one of his favorite

${ }^{1}$ AIM, CEA, CNRS, Université Paris-Saclay, Université Paris Diderot, Sorbonne Paris Cité, 91191 Gif-sur-Yvette Cedex, France 
research topics: modeling and understanding tidal interactions in binary stars and planetary systems and predicting their impact on their orbital and rotational evolutions. This review, after having introduced the general principles of tidal interactions, dissipation, and evolution (Sect. 2), will follow the scientific path of Jean-Paul in this research area for each type of celestial bodies. First, I will review the corner stones he has provided us for the understanding of stellar tides (Sect. 3) and their essential legacy for the study of multiple stars and of stars hosting planetary systems. In particular, I will recall how Jean-Paul was the founder of the theory of the equilibrium and dynamical tides in stars that are now applied in a broad diversity of fluid layers of celestial bodies from the deep envelopes of giant planets to the atmospheres and the oceans of super-Earths. I will also summarize his pioneer efforts to propose robust prescriptions to describe the interactions between turbulence in stellar convective zones and tidal flows. In a second part (Sect. 4), I will summarize the results of his participation to the international Encelade project on the study of the evolution of the system of Saturn and of its orbital architecture, and of giant planets in general. I will highlight our contribution to the coherent modeling of the viscoelastic dissipation of the solid equilibrium tide in their probable dense rocky/icy core, which is a serious candidate to explain recent astrometric measurements of the tidal orbital evolution of the moons of Jupiter and Saturn. In a last part (Sect. 5), I will review the work done by Jean-Paul and Michel Rieutord on the inefficiency of tidal Ekman boundary layers at the free surface of stars and planets. At each step, I will highlight the strong and friendly key collaborations that Jean-Paul has developed with different researchers and $\mathrm{PhD}$ students. I will also summarize the key challenges the community studying tides in stellar and planetary interiors have to address, following the path initiated by Jean-Paul, our friend and mentor.

\section{Tides: General principles}

Stars and planets are submitted to mutual gravitational interactions in close binary stars and planetary systems. When one goes beyond the point-mass approximation for a given body (called the primary), the gravitational force exerted by another body (called the secondary or the companion) is different at its surface and at its centre of mass. A differential gravitational force is applied, which is by definition the tidal force $\left(\mathbf{f}_{\mathrm{T}}\right)$. It can be derived from the tidal potential $\left(U_{\mathrm{T}}\right)$ as:

$$
\mathbf{f}_{\mathrm{T}}=\nabla U_{\mathrm{T}}, \quad \text { where } \quad U_{\mathrm{T}}=\frac{G M_{\mathrm{c}}}{d^{3}} P_{2}[\cos (\mathbf{d} \cdot \mathbf{r})],
$$

where $G$ is the universal gravity constant, $M_{\mathrm{c}}$ the mass of the considered tidal companion treated here as a point-like body with a negligible angular momentum, $d(\mathbf{d})$ the distance (vector) between the centres of mass of the primary and the secondary, $\mathbf{r}$ the current position vector in the primary, and $P_{2}$ the quadrupolar Legendre polynomial. We refer the reader to reference books (e.g. Murray \& Dermott 1999) and articles (Kaula 1962; Mathis \& Le Poncin-Lafitte 2009; Mathis et al. 2013; Ogilvie 2014) for a complete description of the complex mathematical 
formalisms that are used to expand the tidal potential as a function of the orbital Keplerian parameters.

Both in fluid and solid celestial bodies, the tidal force perturbs the hydrostatic equilibrium from which mass redistribution and perturbations of the gravitational potential and pressure result. The mass redistribution in the direction of the companion constitutes the tidal bulge. It induces a large-scale velocity (elastic displacement) in fluid (solid) layers, the so-called fluid (elastic) equilibrium tide (e.g. Zahn 1966a; Remus et al. 2012a; Love 1911; Tobie et al. 2005; Remus et al. $2012 \mathrm{~b}$ ). However, this velocity (displacement), depending on the value of the tidal frequency

$$
\sigma \equiv \ln _{\mathrm{orb}}-m \Omega,
$$

where $n_{\text {orb }}$ and $\Omega$ are the angular velocities of the orbit and of the rotation of the primary, respectively, and $l$ and $m$ are integers, must be completed by fluid (elastic) eigenmodes of oscillation of the primary, the so-called dynamical tide. In fluid bodies, the dynamical tide is constituted by inertial, gravity, Alfvén, and acoustic waves excited by the tidal force. Their restoring forces are the Coriolis acceleration, buoyancy (in convectively stable layers), the magnetic (tension) Lorentz force, and the compressibility, respectively (e.g. Rieutord 2015). In solid bodies, the dynamical tide is constituted by seismic waves (e.g. Alterman et al. 1959; Tobie et al. 2005). In the case where the primary will be frictionless, the equilibrium and dynamical tides will be in phase with the tidal potential and the resulting tidal bulges (one per Fourier mode of the tidal potential) aligned along the line of centres. Moreover, the tidal torque applied on the rotation of the primary and on the orbit vanishes when averaged on a tidal period in this adiabatic situation (e.g. Zahn 1966a). However, this is an ideal situation that never exists and the equilibrium and dynamical tides are submitted to friction mechanisms such as viscosity and heat diffusion. Their kinetic and potential energies are converted into heat that drives a tidal heating that impacts the structure and the evolution of the body. Moreover, because of the dissipation, the direction of tidal bulges has an angle, the tidal angle $\delta$, with the line of centres. This general principle of tidal interactions is summarized in Figure 1.

Therefore, a net tidal torque is applied both on rotation and the orbit. In the simplest case of a circular co-planar system, the torque applied to the spin of the primary can be approximated as (e.g. Zahn 2013):

$$
\Gamma=-\frac{\left(\Omega-n_{\text {orb }}\right)}{t_{\text {friction }}}\left(\frac{M_{\mathrm{c}}}{M_{\mathrm{p}}}\right)^{2} M_{\mathrm{p}} R_{\mathrm{p}}^{2}\left(\frac{R_{\mathrm{p}}}{d}\right)^{6},
$$

where $M_{\mathrm{p}}$ and $R_{\mathrm{p}}$ are the mass and the radius of the primary and $t_{\text {friction }}$ is the time characterizing tidal dissipation. This expression shows the importance of understanding the physics of tidal dissipation in stars and planets from first-principles equations to obtain a robust evaluation of $t_{\text {friction }}$ and predict the evolution of multiple stars and planetary systems and their final states. Moreover, it allows one to identify the crucial importance of the so-called co-rotation radius at which $n_{\text {orb }}=\Omega$. On the one hand, if $n_{\text {orb }}>\Omega$, then the companion migrates inwards 


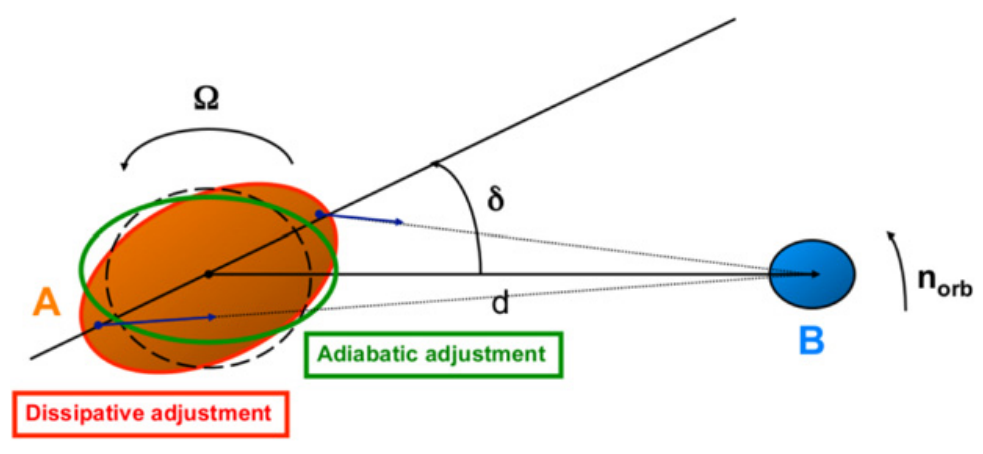

Fig. 1. General principle of tides: a companion (B), which orbits around the primary (A) with an orbital angular velocity $n_{\text {orb }}$ at a distance $d$, generates a tidal bulge because of tidal forces. In an idealized case, where any internal friction in A is ignored, a tidal bulge is created in the direction of the line of centers; this corresponds to an adiabatic tidal adjustment. When tidal friction is taken into account, the response of $\mathrm{A}$ presents a delay, and the tidal bulge becomes misaligned with the tidal angle $(\delta)$; this is the tidal dissipative adjustment. Tidal friction, in the case of stable systems, then tends to synchronize the rotation of $\mathrm{A}(\Omega)$ with the orbital motion $\left(n_{\text {orb }}\right)$ of $\mathrm{B}$, thanks to the tidal torque (Eq. (2.3)).

and the primary's rotation accelerates. On the other hand, if $n_{\text {orb }}<\Omega$, the companion migrates outwards and the primary's rotation slows down. This is the configuration of the Earth-Moon system where the Moon migrates outwards by 3.8 centimetres per year. In the first case $\left(n_{\text {orb }}>\Omega\right)$, if $L_{\text {orb }} \leq 3 L_{\mathrm{p}}$, where $L_{\text {orb }}$ and $L_{\mathrm{p}}$ are the angular momentum of the orbit and of the primary respectively, the companion spirals towards the primary until it is tidally disrupted at the Roche limit (Hut 1980). If $L_{\text {orb }} \geq 3 L_{\mathrm{p}}$, the binary system constituted by the primary and the companion tends, when isolated, towards an equilibrium state where the orbit is circular and the rotational spins and the orbital one are aligned and synchronized. In this framework, the time characterizing dissipation $\left(t_{\text {friction }}\right)$ allows one to predict circularization, alignment and synchronization timescales. Finally, it is usual to express $t_{\text {friction }}$ as a function of a corresponding tidal quality factor $Q$ (MacDonald 1964) inspired by the theory of forced oscillators (e.g. Greenberg 2009). A strong dissipation corresponds to a low-quality factor and friction time (and vice-versa). This quality factor is always combined with the quadrupolar Love number $k_{2}$, defined as the ratio of the self-gravitation potential perturbation and of the tidal potential at the surface of the studied body. It measures its mass concentration. Ogilvie \& Lin 2007 also introduced the modified tidal quality factor $Q^{\prime}=3 Q /\left(2 k_{2}\right)$, which reduces to $Q$ for a homogeneous body. Finally, it is important to point out that each dissipation mechanism has a specific frequency-dependence (i.e. smooth or resonant; we refer to Mathis et al. 2013; Ogilvie 2014, for mathematical formalisms), particularly for the resonant fluid dynamical tide, with important consequences for the dynamical evolution of 

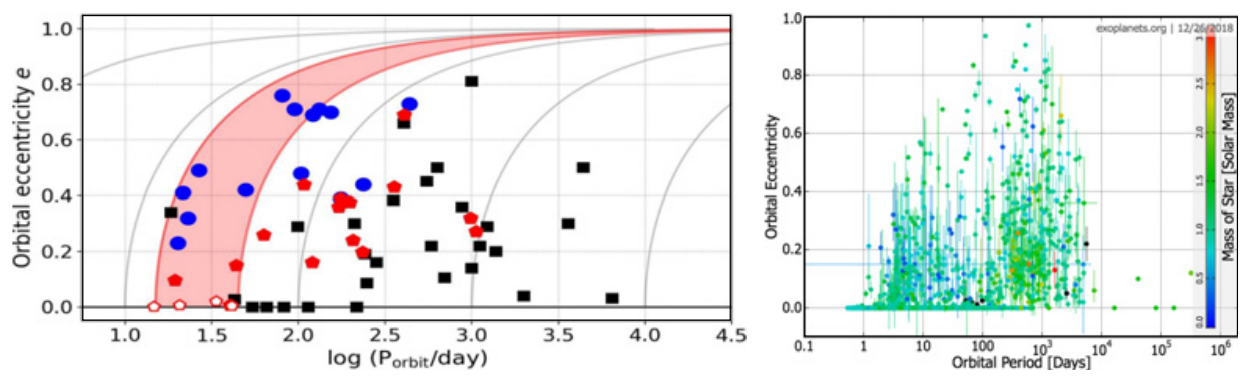

Fig. 2. Diagrams period-eccentricity of binary stars hosting a red giant component (left panel; in black the systems reported in Verbunt \& Phinney (1995) and in red and blue Kepler seismic binaries (Beck et al. 2018, and references therein); taken from Beck et al. 2018, courtesy Monthly Noticies of the Royal Astronomical Society) and of already discovered exoplanets for which orbital properties are available (right panel; computed using the database exoplanets.org; the mass of the host stars is indicated in color scale).

binary and multiple systems (e.g. Zahn \& Bouchet 1989; Witte \& Savonije 2002; Efroimsky \& Lainey 2007; Auclair-Desrotour et al. 2014). Each tidal dissipation mechanism in stars and planets should thus be carefully examined.

\section{Stellar tides}

On one hand, binary stars constitute an important population (approximatively $50 \%$ of main-sequence stars) (Duquennoy \& Mayor 1991; Sana \& Evans 2011). They provide us precise knowledge of stellar masses and constrains on the internal structure of stars thanks to the apsidal motion induced by tides. A deep understanding of their tidal dynamics is thus very important for the theory of stellar evolution (see also the contribution by Lionel Siess). In this framework, several studies focusing on their circularization (and synchronization) period (i.e. the orbital period below which their orbits are circularized) are probing for different stellar masses and evolutionary stages the tidal friction mechanisms in stars (Koch \& Hrivnak 1981; Giuricin et al. 1984; Mathieu \& Mazeh 1988; Mermilliod et al. 1992; Duquennoy et al. 1992; Verbunt \& Phinney 1995; Claret \& Cunha 1997; Latham et al. 2002; North \& Zahn 2003; Mathieu et al. 2004; Meibom \& Mathieu 2005; Meibom et al. 2006; Khaliullin \& Khaliullina 2010; Beck et al. 2018, the left panel of Fig. 2, and the contributions by Paul Beck, Tamas Borkovits, and János Sztakovics).

On the other hand, from 51 Pegasi-b, the first discovered exoplanet (Mayor \& Queloz 1995) to the recently detected Trappist-1 system (Gillon et al. 2017), a large number of the known exoplanets orbit very close to their host stars. In such compact configurations, star-planet tidal interactions, and more specifically the dissipation of tides in stars, play a key role to shape systems' orbital architecture. A first signature of its action is the small orbital eccentricity of systems with small 
orbital periods (e.g. $<10$ days for Jupiter-mass exoplanets; see also the right panel of Fig. 2) and the wide range of eccentricities observed for longer periods. Another potential example is the low (high) spin-orbit inclination observed around stars with a thick (thin) convective envelope (e.g. Winn et al. 2010; Albrecht et al. 2012), which possibly hosts strong (weak) tidal dissipation. Mazeh et al. (2015) showed however that this phenomenon is also observed for long period systems (above 50 days), where tides should not be efficient, that challenges tidal theories. These observations have been used to constrain and calibrate tidal dissipation in stars hosting planets (e.g. Jackson et al. 2008; Husnoo et al. 2012; Hansen 2012).

Combining observations in tidally interacting binary stars and in exoplanetary systems allows us to identify that tidal dissipation in stars varies strongly with stellar mass, age, and rotation, while several friction mechanisms should be involved. This may have major consequences for exoplanetary systems, which have been observed around a large diversity of host stars. A detailed study of the three components of tidal dissipation in stellar interiors: the dissipation of the equilibrium tide, the dissipation of tidal gravito-inertial waves in stably stratified radiative zones, and the dissipation of tidal inertial waves in their convective zones, and of their possible nonlinearities, resonances, and boundary layers, is thus needed.

\subsection{The equilibrium tide and the convective turbulent friction}

The founder of the theory of the equilibrium tide in stellar interiors was Jean-Paul during his $\mathrm{PhD}$ thesis. He computed its velocity field (Zahn 1966a, see also Remus, Mathis and Zahn 2012 (Fig. 3 - left panel)) and its dissipation by the friction applied by turbulence and the diffusion of heat in convective and radiative zones, respectively (Zahn 1966b,c). He demonstrated that the dissipation of the equilibrium tide is efficient in the convective envelope of low-mass stars (from M- to F-type stars), but it is negligible in the convective core of intermediatemass and massive stars (from A- to O-type stars) and in stellar radiation zones (Zahn 1977, and Fig. 4 - bottom panel). For the case of the convective envelope of low-mass stars, it was thus important to propose a robust modelling of the turbulent friction applied by convection on the velocity field of the equilibrium tide. Assuming a space-scale separation between convective eddies and the large-scale flow of the equilibrium tide and the isotropy of turbulence, which corresponds to neglect the impact of rotation and magnetic fields on convection, Zahn (1966b) proposed a mixing-length model based on characteristic velocity $\left(V_{\mathrm{c}}\right)$ and length scales $\left(l_{\mathrm{c}}\right)$ for convection. Moreover, Zahn (1966b) identified that in the case of tidal periods $\left(P_{\mathrm{t}}=2 \pi / \sigma\right.$, where $\sigma$ is the tidal frequency defined above) short in comparison to the convective turnover time (the so-called "rapid" tides regime), the turbulent friction becomes less efficient because convection has not enough time to apply an efficient damping to tidal flows. Zahn (1966b) proposed that this loss of efficiency scales linearly with the ratio between the tidal period $\left(P_{\mathrm{t}}\right)$ and the characteristic convective time $\left(P_{\mathrm{c}}=l_{\mathrm{c}} / V_{\mathrm{c}}\right)$; Goldreich \& Keeley (1977) proposed a scaling proportional to $\left(P_{\mathrm{t}} / P_{\mathrm{c}}\right)^{2}$ based on the Kolmogorov theory for turbulence. These 

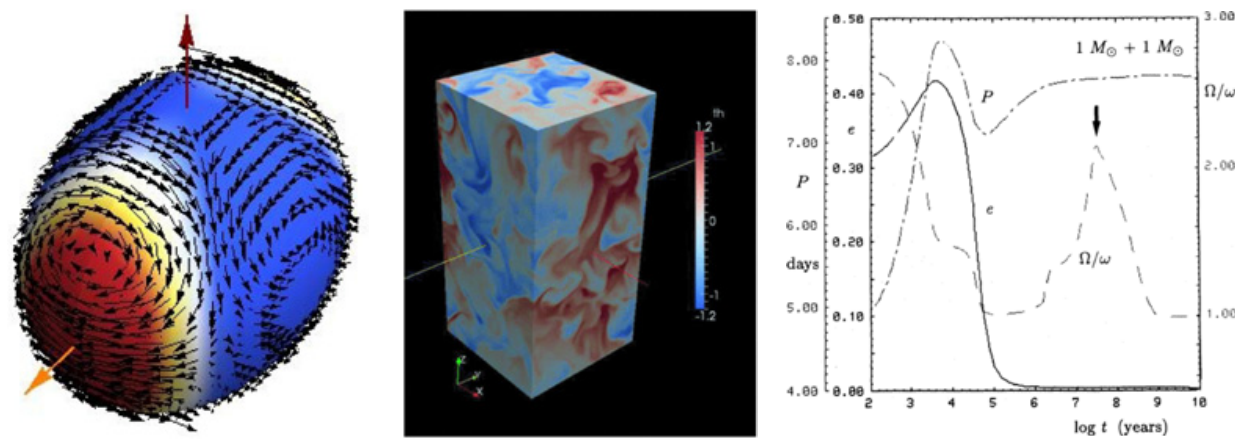

Fig. 3. Left: velocity field of the equilibrium tide at the surface of a convective star or planet represented by black arrows. The red and orange arrows represent the rotation axis and the direction of the companion, respectively. The colors from blue to red give the intensity of the tidal potential (taken from Remus et al. 2012a, courtesy Astronomy \& Astrophysics). Middle: direct numerical simulation of the nonlinear interactions between the convective turbulence and an oscillatory tidal equilibrium flow (taken from Ogilvie \& Lesur (2012), courtesy Monthly Noticies of the Royal Astronomical Society). Right: orbital and rotational evolutions of a $1 M_{\odot}+1 M_{\odot}$ binary star due to the turbulent dissipation of the equilibrium tide; in solid line, the eccentricity $(e)$ as a function of time $(t)$; in dashed-dotted line, the orbital period $(P)$ as a function of time; in dashed line, the synchronism of the system given by the ratio $\Omega / \omega(\Omega$ and $\omega$ being the rotation of the primary and the orbital frequency, respectively) as a function of time (taken from Zahn \& Bouchet 1989, courtesy Astronomy \& Astrophysics).

two propositions have been tested using direct numerical simulations of convective turbulence submitted to periodic shears and are still debated (e.g. Penev et al. 2007; Ogilvie \& Lesur 2012, (Fig. 3 - middle panel) and the contribution by Gordon Ogilvie). More recently, Mathis et al. (2016) examined the impact of rotation. Using scaling laws derived theoretically by Stevenson (1979) and verified by direct numerical simulations of turbulent convective rotating flows (Barker et al. 2014), they demonstrated how rotation weakens convection efficiency and the related turbulent friction. The dissipation of the equilibrium tide in rapidly rotating stars should thus be less efficient than in slow rotators. When applied and confronted to observations, this tidal friction mechanism can successfully explain the orbital state of binary low-mass stars for ages below 1 Gyr (Zahn \& Bouchet 1989; Zahn 2013, and references therein; see Fig. 3 - right panel) because of the efficient orbit circularization during the Pre-Main-Sequence (hereafter PMS) while stars have a large radius and a deep convective envelope. When taking into account the structural evolution of stars, it also predicts well the orbital state of binary stars hosting a red giant component (Verbunt \& Phinney 1995; Beck et al. 2018). However, for late-type binaries older than $\sim 1 \mathrm{Gyr}$, the equilibrium tide is not strong enough to explain the observations (e.g. Meibom \& Mathieu 2005; Zahn 2013) and the dynamical tide should thus be introduced. 


\subsection{The dynamical tide}

\subsubsection{In stellar radiation zones}

Stellar radiation zones are the seats of tidal waves. Because of their stable entropy stratification, combined with stellar rotation, tidal waves become gravito(-inertial) waves (e.g. Zahn 1975; Ogilvie \& Lin 2007; André et al. 2017). Their dynamics is therefore driven simultaneously by buoyancy and the Coriolis acceleration (see Fig. 4 - top panel). They are generally excited near the convection/radiation boundaries and dissipated (in the linear theory; see Sect. 3.3 for nonlinear mechanisms) by heat diffusion.

In the case of early-type stars, this dissipation mechanism is efficient since the thermal diffusivity is important close to the surface where tidal gravity waves are propagative and can be completely damped for low-frequency ones. Goldreich \& Nicholson (1989) demonstrated in the case of a radially differentially rotating star that this will lead to the synchronization of stellar layers from the surface to the centre. For these stars, Zahn (1977) demonstrated that this dissipation of pure gravity waves dominates the dissipation of the equilibrium tide (see Fig. 4 - bottom panel). This mechanism can explain the circularization state of early-type binaries (e.g. North \& Zahn 2003) for $a / R<4$ (where $a$ and $R$ are the orbital semi-major axis and the stellar radius, respectively). However, for binaries for which $a / R>4$, a fraction of stars is also circularized calling for another, more efficient, dissipation mechanism. Rocca $(1987,1989)$ introduced rotation through the action of the Coriolis acceleration and demonstrated it increases tidal dissipation (see also e.g. Papaloizou \& Savonije 1997; Savonije \& Witte 2002, and references therein). More specifically, Witte \& Savonije (1999a,b) showed that because of the action of the Coriolis acceleration, retrograde and prograde tidal modes exert torques of opposite sign that may trap an eccentric binary in a resonant state where dissipation is stronger. This may explain the circularized population of binaries for which $a / R>4$.

In the case of low-mass stars with a radiative core (see Fig. 4 - middle left panel), heat diffusion acting on tidal gravity waves is less efficient since they are evanescent in the convective envelope where the thermal diffusivity is the highest. Terquem et al. (1998) and Goodman \& Dickson (1998) demonstrated that the dissipation of these waves can compete, at resonant frequencies, with the turbulent dissipation of the equilibrium tide in the convective envelope. This result has been generalised by Ivanov et al. (2013) and Chernov et al. (2013) in the case of tidal gravito-inertial waves. Moreover, when looking at the orders of magnitude of the corresponding tidal quality factor computed by Ivanov et al. (2013), one sees it can be of the same order of magnitude that the one computed for tidal inertial waves (Mathis 2015a, see the next subsection). As in the case of early-type stars, including rotation with the associated potential resonant lockings (Ogilvie \& Lin 2007; Witte \& Savonije 2002) allows to get a higher tidal dissipation rate, but still not efficient enough to explain the circularization state of old late-type binaries (Mathieu et al. 2004). In addition, gravito(-inertial) waves' nonlinear breaking near the center of solar-type stars (for stellar masses below which there 


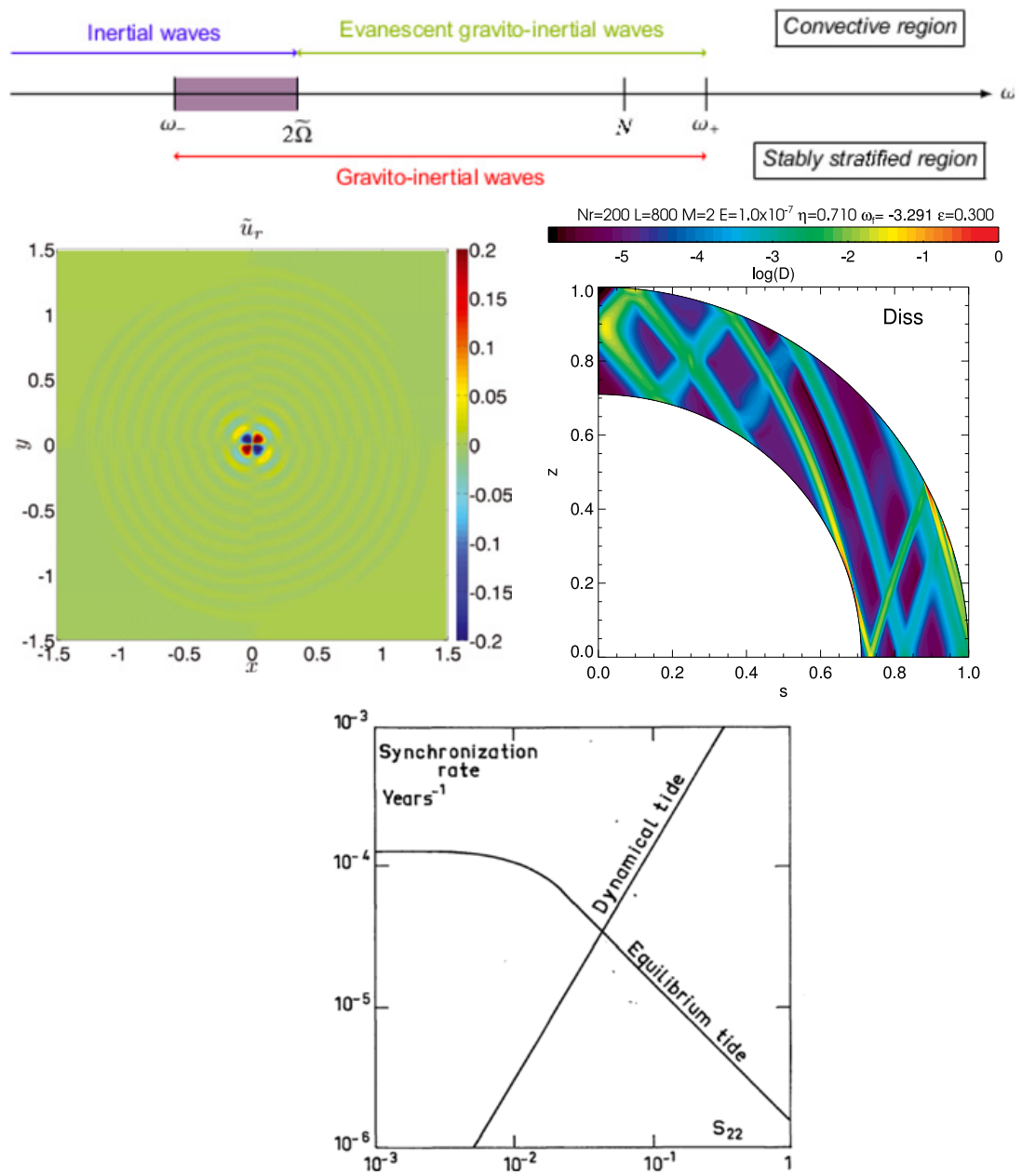

Fig. 4. Top: frequency spectrum of tidal (gravito-)inertial waves and their behavior in convective and stably stratified regions $\left(\omega_{ \pm}\right.$are their maximum and minimum frequencies, $N$ is the Brunt-Väisälä frequency, and $\widetilde{\Omega}$ the effective rotation; taken from André et al. (2017), courtesy Astronomy \& Astrophysics). Middle-left: radial component of a tidal gravity wave propagating in the equatorial plane of the radiative core of a $1 M_{\odot}$ star (taken from Barker 2011, courtesy Monthly Noticies of the Royal Astronomical Society). Middle-right: viscous dissipation of the kinetic energy of a tidal inertial wave attractor in the convective envelope of a solar-type star with the solar latitudinal differential rotation (see also Guenel et al. 2016a). Bottom: Comparison of the efficiency of the equilibrium and dynamical tides for a $5 M_{\odot}$ star through their intrinsic synchronization rates as a function of the normalized tidal frequency $s_{22}=2(\Omega-\omega) /\left(R^{3} /(G M)\right)^{1 / 2}(\Omega$ is the rotation of the star, $\omega$ the orbital frequency, $R$ and $M$ the stellar radius and mass, and $G$ the universal constant of gravity; taken from Zahn (1977), courtesy Astronomy \& Astrophysics). 
is no convective core) provides an interesting avenue to explain the survival of "hot" Jupiters (e.g. Barker \& Ogilvie 2010; Guillot et al. 2014).

Finally, the theory of stellar tidal gravito-inertial waves have an important growing legacy for the study of the dissipation of these waves in planetary interiors, from the atmospheres of gaseous giant and telluric (exo-)planets (e.g. Ogilvie \& Lin 2004; Gu \& Ogilvie 2009; Arras \& Socrates 2010; Auclair-Desrotour et al. 2017; Auclair-Desrotour \& Leconte 2018, and the contribution by Pierre-Auclair Desrotour) to the potential deep oceanic layers of telluric exoplanets and icy moons (e.g. Auclair-Desrotour et al. 2018).

\subsubsection{In stellar convection zones}

When the orbital period $\left(P_{\text {orb }}\right)$ is such that $P_{\text {orb }}>1 / 2 P_{\mathrm{s}}$ in a coplanar circular system, where $P_{\mathrm{s}}$ is the rotational period of the star, and assuming as a first step a uniform rotation, tidal inertial waves are excited in stellar convective regions (Bolmont \& Mathis 2016). In the more general case one has $-2 \Omega<\sigma<2 \Omega$, where $\sigma$ is the tidal frequency defined in Equation (2.2).

In the case of convective cores (for stars whose masses are above $\approx 1.1 M_{\odot}$, where $M_{\odot}$ is the solar mass) and fully convective M-type stars (with masses $<0.45 M_{\odot}$ ), tidal waves propagate in the full sphere. Therefore, they are able to form regular normal inertial modes (e.g. Bryan 1889; Braviner \& Ogilvie 2014). The turbulent friction already described above in the case of the equilibrium tide, drives the dissipation of these modes forced by tidal forces. It leads to a resonant frequency-dependent tidal dissipation, as in the case of a classical forced oscillator (e.g. Greenberg 2009). At the level of a resonance, the dissipation of tidal inertial waves can be larger by possibly several orders of magnitude than the dissipation of the equilibrium tide (e.g. Braviner \& Ogilvie 2015), depending on the value of the effective turbulent viscosity (Auclair Desrotour et al. 2015); the weaker the viscosity, the stronger the resonances. These resonances strongly impact the orbital and the rotational dynamics (e.g. Witte \& Savonije 2002; Auclair-Desrotour et al. 2014). Note that this mechanism has also been studied in the case of coreless giant planets with similar conclusions (Wu 2005a,b).

The case of stars with a convective envelope surrounding a radiative core (with masses from $\approx 0.45$ to $\approx 1.4 M_{\odot}$ ) is much complex. In fact, in the case of inertial waves propagating in spherical shells, the cylindrical geometry imposed by the Coriolis acceleration conflicts with the spherical geometry of the container and the problem becomes ill posed in the limit of vanishing viscosity (Rieutord \& Valdettaro 1997). In this case, some of the waves focus through their reflexions on the radiative core and on the stellar surface on limit cycles, the so-called waves attractors (e.g. Ogilvie 2005, and Fig. 4 - middle right panel). Along these structures, the velocity field is sheared and the resulting resonant dissipation (see Fig. 5- left panel) becomes stronger than in the case of regular normal inertial modes, which propagate in a full sphere. This provides a potential efficient mechanism of tidal dissipation in the convective envelope of low-mass stars hosting planetary systems (Ogilvie \& Lin 2007; Barker \& Ogilvie 2009) and in giant planets 

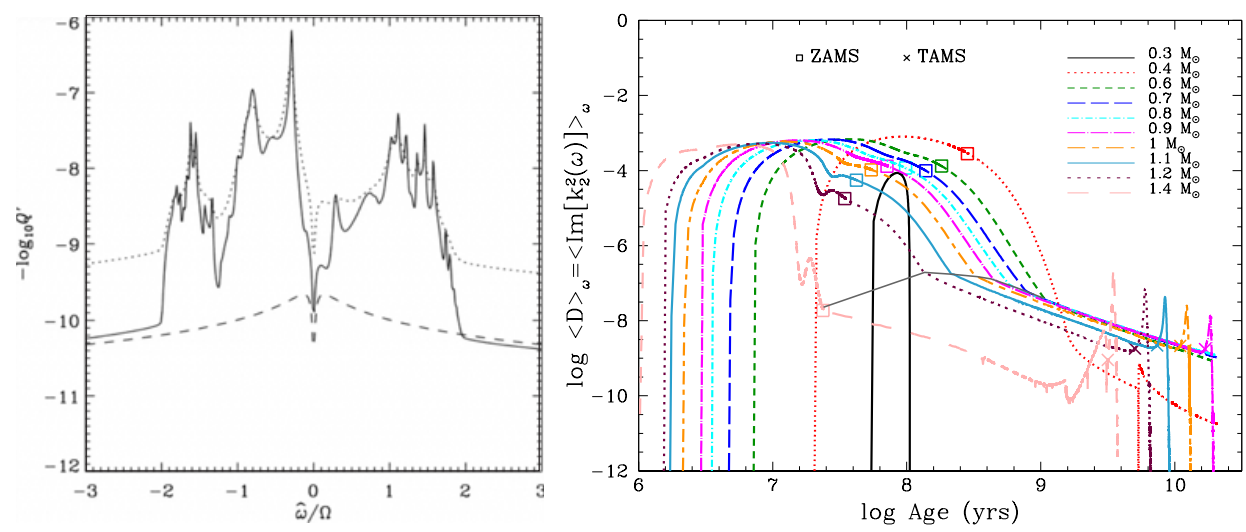

Fig. 5. Left: frequency-dependence of the viscous dissipation of tidal inertial waves (expressed through a tidal quality factor $Q^{\prime}$ ) propagating in the convective envelope of a uniformly rotating $1 M_{\odot}$ star with a spin period of 10 days (taken from Ogilvie \& Lin 2007, courtesy The Astrophysical Journal); $\widehat{\omega} \equiv \sigma$ is the tidal frequency. Right: Variation of the frequency-averaged dissipation of tidal inertial waves in the convective envelope of low-mass stars along their evolution (taken from Gallet et al. 2017, courtesy Astronomy \& Astrophysics).

with a central dense core (Ogilvie \& Lin 2004). As demonstrated by Goodman \& Lackner 2009 and Ogilvie 2013, who considered the frequency-averaged dissipation, this mechanism has a strong dependence on the internal structure (i.e. the radius and the mass aspect ratios between the convective envelope and the radiative core) and the rotation of stars. Using grids of modern stellar structure and evolution models, Mathis (2015b); Gallet et al. 2017, and Bolmont et al. (2017) demonstrated how the dissipation varies over several orders of magnitude as a function of stellar mass, age, rotation, and metallicity (see Fig. 5 - right panel and the contribution by Florian Gallet). These works demonstrated that the frequency-averaged tidal dissipation is maximal for intermediate radius and mass aspect ratios $\left(\alpha=R_{\mathrm{c}} / R_{\mathrm{s}}=\alpha_{\max }=0.571\right.$ and $\beta=M_{\mathrm{c}} / M_{\mathrm{s}}=\beta_{\max }=0.501$, where $R_{\mathrm{c}}\left(M_{\mathrm{c}}\right)$ and $R_{\mathrm{s}}\left(M_{\mathrm{s}}\right)$ are the radius(mass) of the radiative core and of the star, respectively). In this regime, the radius aspect ratio is such that wave attractors (and critical latitudes phenomena) can develop while the involved volume is important enough to get an important dissipation. These are the reasons why dissipation should be weak both in main-sequence fully-convective M-type and F-type stars because of the absence of wave attractors in the first case and of the very small thickness of the convective envelope in the second one. Drawing the evolutionary tracks of low-mass stars from M-type to F-type stars in the $(\alpha, \beta)$ plane, they reach the state of maximum averaged dissipation during the premain-sequence because of their structural evolution due to the formation and growth of the radiative core. During their main-sequence, the structure of lowmass stars becomes almost constant. The frequency-averaged dissipation is then 


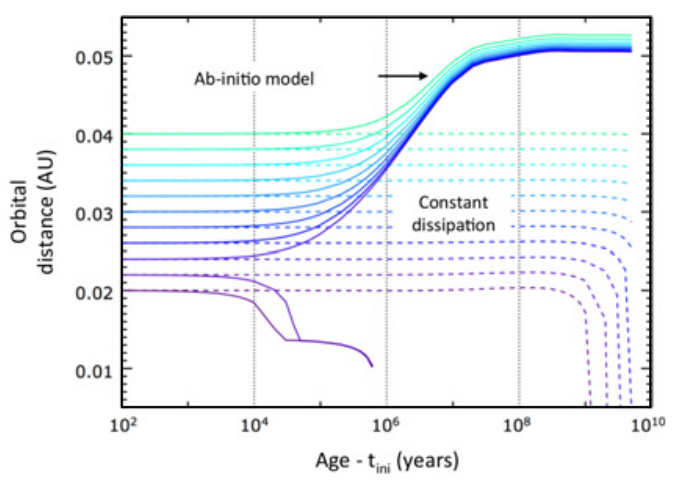

Fig. 6. Evolution of the orbital semi-major axis (in Astronomical Units) of a Jupitermass close-in "hot" Jupiter orbiting a $1 M_{\odot}$ star assuming a constant dissipation in the star along its evolution (in dashed lines) and taking into account the evolution of tidal dissipation in its rotating convective envelope computed using the ab-initio model established in Ogilvie (2013) and Mathis (2015b) (in solid lines) (taken from Bolmont \& Mathis 2016, courtesy Celestial Mechanics and Dynamical Astronomy). The initial time, after the dissipation of the proto-planetary disk, is $t_{\text {ini }}$.

driven by the decrease of their rotation because of the braking applied by stellar winds. When leaving the main-sequence, the dissipation first increases again during the subgiant phase because of the contraction of the radiative core and the extension of the convective envelope until $\alpha \rightarrow \alpha_{\max }$ and $\beta \rightarrow \beta_{\max }$. After, it decreases when the star becomes a red giant with a large convective envelope and a small convective envelope (i.e. $\alpha \rightarrow 0$ and $\beta \rightarrow 0$ ); the situation is then closer to a full sphere configuration with a weak dissipation of tidal inertial waves.

The resulting tidal orbital migration of short-period planets is strongly different than those predicted by usual models where tidal dissipation in a star is assumed to stay constant all along its evolution (Bolmont et al. 2012; Bolmont \& Mathis 2016; Gallet et al. 2017; Bolmont et al. 2017; Gallet et al. 2018; Benbakoura et al. 2019, Fig. 6 and the contribution by Emeline Bolmont). It allows us for instance to explain some observational trends in the orbital distribution of exoplanets like the lack of "hot"-Jupiters around fast rotating stars (e.g. McQuillan et al. 2013; Teitler \& Königl 2014). However, Damiani \& Mathis (2018) demonstrated that this frequency-averaged dissipation, when applied to the obliquity tide (Lai 2012), is too strong to explain the observed dichotomy in spinorbit inclinations (Winn et al. 2010; Albrecht et al. 2012); indeed, it damps these inclinations for all low-mass stars. This shows that either this modelling should be improved by taking into account the frequency-dependence of the tidal torque, the realistic density profile in stars instead of simplified bi-layer stellar models with averaged densities for the radiative core and the convective envelope that may overestimate the dissipation (see Fig. 10 in Ogilvie 2013), supplementary physical 
ingredients (see the next section), or that other mechanisms should be invoked (e.g. Lai et al. 2011; Batygin 2012; Rogers \& Lin 2013; Fielding et al. 2015; Li \& Winn 2016).

\subsection{Current challenges}

\subsubsection{Nonlinearities and resonances}

In stellar interiors, non-linearities can develop both in convective regions and in stably stratified radiation zones. First, high-amplitude tidal gravity waves excited by massive close companions can break at the centre of low-mass stars with a central radiative core (e.g. Barker \& Ogilvie 2010). The reason for such a behaviour is the decreasing stratification and the geometric focusing of waves at their centre (Press 1981). Next, in the case of important tidal deformations, i.e. for short-period systems, large-scale tidal non-wave like flows can become unstable to the so-called elliptic instability (Kerswell 2002). Then, a given (gravito-)inertial wave can excite another one, leading to the instability, turbulence, and finally dissipation (for a complete review, we refer the reader to Le Bars et al. 2015, and references therein and to the contributions by Adrian J. Barker and Benjamin Favier). In hydrodynamical simulations, Barker \& Lithwick (2013) and Barker (2016) identified the key role of the differential rotation driven by the instability. This zonal flow acts to saturate its cause, the instability, leading to intermittent dissipation. In presence of a weak magnetic field or another mechanism that damps differential rotation, turbulence is sustained leading to a stronger dissipation (Barker \& Lithwick 2014).

\subsubsection{Zonal flows and magnetic fields}

Finally, in stellar (and planetary) interiors, internal mechanisms, like for instance convection, can sustain differential rotation (see Mathis 2013; Brun et al. 2015, and references therein). Then, tidal inertial and gravito-inertial waves are modified by the imposed shear (Mathis 2009; Baruteau \& Rieutord 2013; Guenel et al. 2016a; Mirouh et al. 2016). Particularly, co-rotation layers, where the local angular velocity is proportional to the orbital frequency, play a key role in tidal dissipation both in radiative (Goldreich \& Nicholson 1989) and in convective regions (Favier et al. 2014; Guenel et al. 2016b) because of wave-mean flows interactions. In forthcoming works, it would be important to compare the strength of zonal flows generated by tidal waves (e.g. Favier et al. 2014) and internal mechanisms, respectively. In addition, stars (and planets) are magnetized objects and the impact of stellar (planetary) magnetic fields on tidal waves (e.g. Wei 2016; Lin \& Ogilvie 2018; Wei 2018) should be taken into account all along their evolution (Astoul et al. 2018). Potential tidally-induced modification of stellar magnetic fields should also be examined carefully (e.g. Cébron \& Hollerbach 2014). Finally, comparison of tidal effects and direct magnetic star-planet interactions should be evaluated as a function of stellar and planetary properties (Strugarek et al. 2017). 


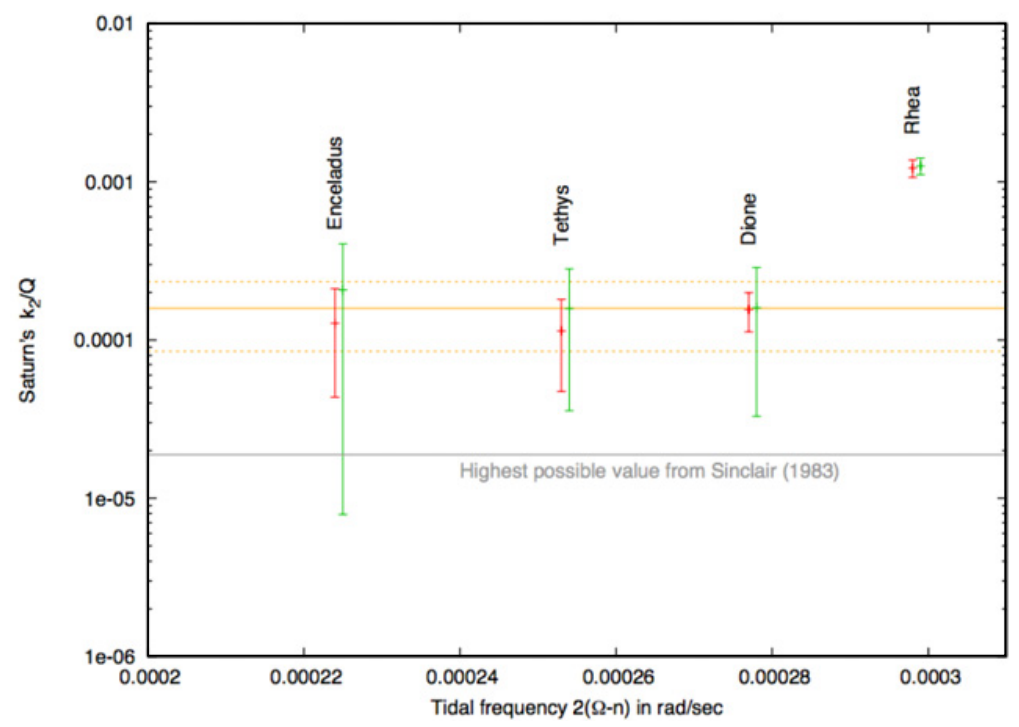

Fig. 7. Tidal dissipation in Saturn, measured thanks to high-precision astrometry, at the tidal frequencies of Enceladus, Tethys, Dione, and Rhea (taken from Lainey et al. 2017, courtesy Icarus). The dissipation is expressed as a function of the second-order Love number $k_{2}$, which evaluates the amplitude of the hydrostatic tidal deformation, and the tidal quality factor $Q$.

\section{Tides in giant planets}

\subsection{A stronger dissipation than expected in Saturn and Jupiter: The Encelade team adventure}

During the last decade, new discoveries about Jupiter, Saturn, and their systems invited us to revisit the current estimations of tidal dissipation in gaseous giant planets of our solar system. Using high precision astrometry, Lainey et al. (2009, 2012, 2017); Polycarpe et al. (2018) demonstrated that tidal dissipation in Jupiter and Saturn are one order of magnitude higher than what was computed initially by Goldreich \& Soter (1966) (see also Sinclair 1983) as shown in Figure 7 (i.e. a tidal quality factor $\sim 10^{3}$ instead of $\sim 10^{4}$ for Saturn). Moreover, the dissipation in Saturn has a complex variation as a function of the tidal frequency: we observe a weak (and potentially smooth) dependence on the frequency for Encelade, Tethys, and Dione, and a more important dissipation for Rhea (and Titan). We recall that the initial predictions for the tidal quality factors of Jupiter and Saturn were computed by Goldreich \& Soter (1966) who solved the evolution equation for the semi-major axis of the moons assuming a frequency-independent tidal dissipation rate, constant over the evolution, in order to place them at their current positions at the age of the solar system. Observing a stronger dissipation thus potentially 
also requests revisions of our understanding of the formation and evolution of the systems of giant planets in the Solar (and exoplanetary) system(s) (Charnoz et al. 2011) and of tidal dissipation in their interiors.

These results are also of major interest for exoplanetary systems since gaseous giant planets have been the first type of discovered exoplanets. Indeed, the discovery of 51 Pegasi b by Mayor \& Queloz 1995 opened the path to the exploration of giant planets outside our Solar system. The occurrence of gaseous giant planets close to their host stars, the so-called "hot" Jupiters, then demonstrated, as already pointed out before, that the orbital architecture of our solar system is not common, sustaining new challenging questions about the formation, the dynamical evolution, and the stability of exoplanetary systems. In this context, the study of tidal (and magnetic) star-planet interactions between low-mass stars and close giant planets also came on the forefront. For example, tidal interactions between a host star and orbiting giant exoplanets were proposed as a candidate to explain the "bloated" hot Jupiter phenomenon (Baraffe 2005). The proposed idea is that important tides are able to sustain internal heating in giant exoplanets, with sufficiently large amplitude, to modify their internal structure with an increase of their radius. Another important but still unsolved question is to get a deep understanding of the orbital distribution of hot Jupiters around their host stars for which both tidal dissipation in the stars and the planets can play a key role. Let us finally point that these major questions are among the key objectives of ongoing and forthcoming major space missions like CHEOPS, TESS, JWST, PLATO, and ARIEL (Benz et al. 2017; Ricker et al. 2015; Lagage 2015; Rauer et al. 2014; Tinetti et al. 2018) and ground-based instruments like SPIRou (Moutou et al. 2015).

The importance of the results obtained by Valéry Lainey and his collaborators and the need for different and complementary expertises pushed him to create the international ENCELADE team in 2012. The team was involving experts in ground- and space-based astrometry, in solar and exoplanetary systems' planetology, in celestial mechanics, and finally in stellar and planetary internal structures among who Jean-Paul and myself (see Fig. 8). It was first supported and funded by the Université Pierre et Marie Curie in Paris while it is now an ISSI team (we refer the reader to its website for more details: http://www.issibern.ch/teams/ saturnastrometry). The project also led Jean-Paul, Valéry and myself to supervise the $\mathrm{PhD}$ Thesis of Françoise Remus on tides in giant planets and exoplanetary systems.

\subsection{A new picture to understand tidal dissipation in giant planets interiors}

\subsubsection{The stratified internal structure of gaseous giant planets}

As explained before when describing the general principles of tidal dissipation, the internal structure of stars and planets strongly impacts dissipative mechanisms acting on tidal flows in gaseous or liquid layers and tidal displacements in solid rocky or icy regions. This is of major importance in the case of multi-layered 


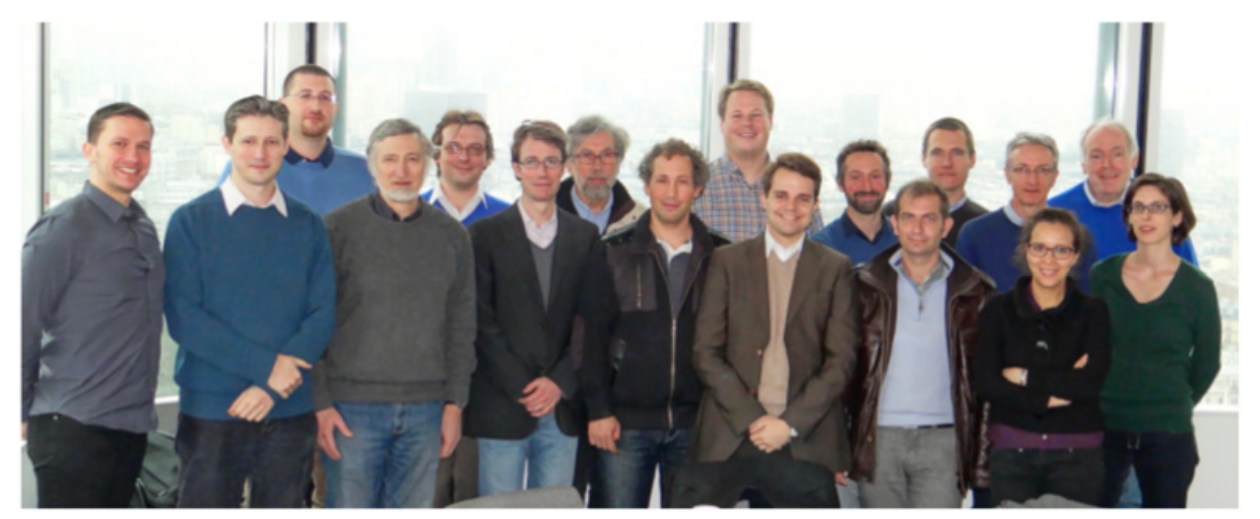

Fig. 8. The Encelade team. First line from left to right: Radwan Tajeddine, Valéry Lainey, Jean-Eudes Arlot, Vincent Robert, Özgür Karatekin, myself, Sébastien Charnoz, Maryame El Moutamid, Françoise Remus; second line from left to right: Kévin Baillé, Christophe Le Poncin-Lafitte, Jean-Paul, Benoît Noyelles, Nicolas Rambaux, Stéphane Renner, Nick Cooper, Carl Murray.

differentiated bodies such as planets for which a robust and detailed knowledge of the internal structure would be ideally requested. In the case of gaseous giant planets, this constitutes one of the important challenge for the evaluation of tidal dissipation.

In a "standard" model, gaseous giant planets are constituted, going from their core to their surface, by a dense core constituted of rocks and ices, surrounded by a deep convective fluid envelope on the top of which lies a potentially stably stratified atmosphere because of the irradiation by the host star (e.g. Guillot 1999; Baraffe et al. 2014, and Fig. 9). In this picture, the presence of a solid core is requested from the core-accretion scenario (Pollack et al. 1996), which allows us to explain the formation of giant planets with a deep gaseous envelope constituted for its major part of hydrogen and helium. However, this simple picture may have to be revised because of the chemicals mixing that may modify the radial structure of giant planets. Indeed, some heavy elements composing the core can be thermodynamically unstable in the conditions of temperature and pressure that reign in giant planets (e.g. Wilson \& Militzer 2012; Mazevet et al. 2015). This can lead to the melting of the core in the fluid envelope. Recent constraints provided by the space mission JUNO (Bolton et al. 2017) using the precise measurements of planetary gravitational moments suggest that this can be the case in the center of Jupiter (Wahl et al. 2017). Moreover, planetesimals may have impacted giant planets in the early phase of their evolution before the proto-planetary disc clears (Stevenson 1985). Finally, a helium rain may occur in the transition region between molecular and metallic hydrogen and helium layers (Salpeter 1973; Stevenson 1975). These three situations may sustain stabilizing compositional gradients in the deep convective envelopes of giant planets. They may in turn trigger 


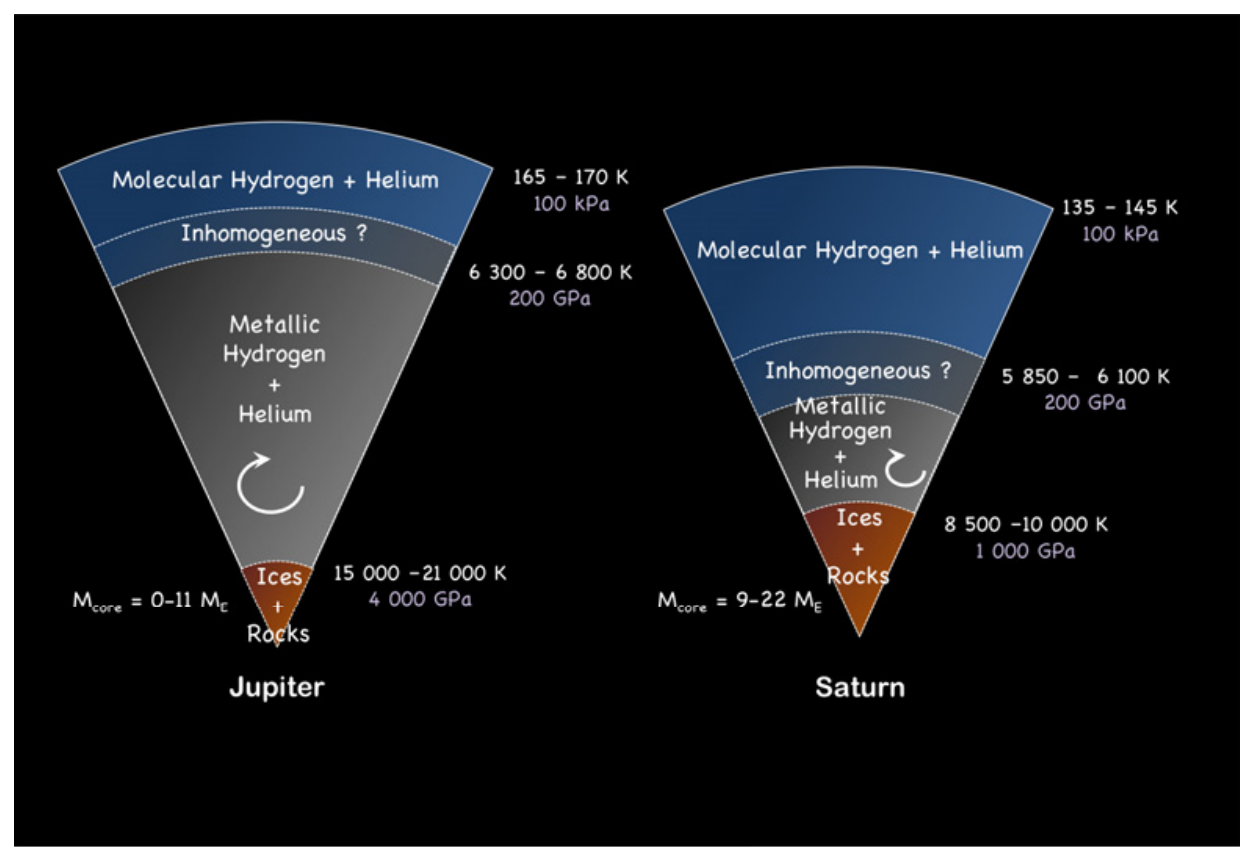

Fig. 9. Internal structure of the gaseous giant planets of our solar system, Jupiter and Saturn (adapted from Guillot 1999).

"non-standard" hydrodynamical mixing processes such as double diffusive convection (e.g. Leconte \& Chabrier 2012; Nettelmann et al. 2015, and Fig. 11) where the unstable entropy gradient competes with a stabilizing chemical gradient. Parts of the fluid envelope can be constituted by a succession of convective layers separated by stably stratified interfaces. New giant planets models have been built including these mechanisms and they have been proposed to explain successfully the excess of Saturn's luminosity in infrared (Leconte \& Chabrier 2013) and to play a role in explaining the abnormally large radius of some hot Jupiters (Chabrier \& Baraffe 2007).

\subsubsection{The dissipation in the dense core of giant planets}

A solid core is therefore needed to ensure the accretion and the stability of a deep gaseous envelope (Pollack et al. 1996). Then, in addition of constituting a boundary for tidal flows in the envelope (i.e. the equilibrium and the dynamical tides reviewed in the previous stellar section), it constitutes on its own a potential strong source of tidal dissipation. The first author who identified the possible reservoir of dissipation constituted by the dense core of giant planets was Dermott (1979). He studied the solid equilibrium tide in homogeneous cores and demonstrated how 

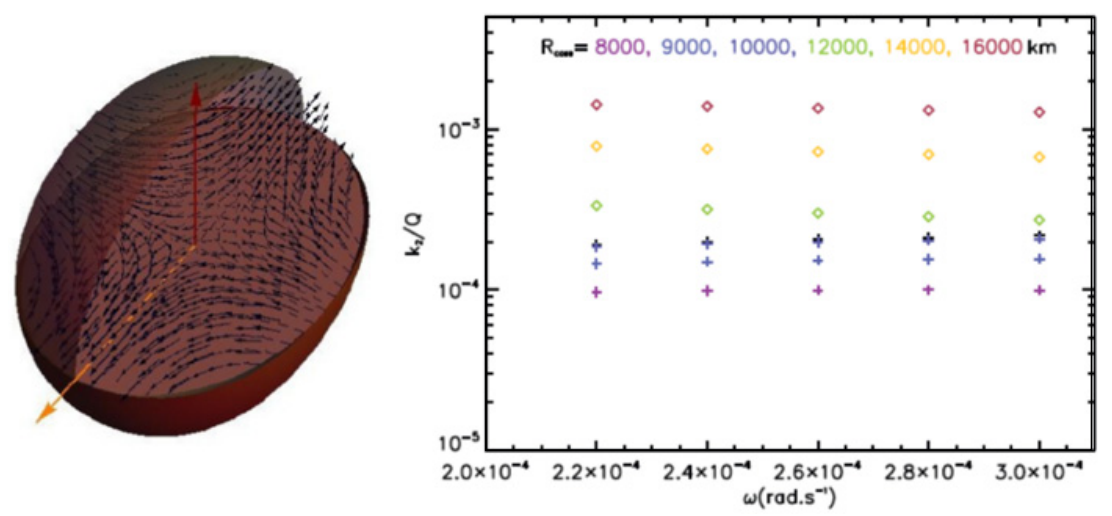

Fig. 10. Left: elastic equilibrium tide in the rocky/icy core of a giant planet (taken from Remus et al. 2012b, courtesy Astronomy \& Astrophysics). The red and orange arrows are the rotation axis and the direction of the companion, respectively. Right: Tidal dissipation in the solid core of Saturn, computed for different values of its radius as a function of the tidal frequency, taking into account its density radial stratification, for a viscosity $\eta=10^{15}$ Pa.s (taken from Lainey et al. 2017, courtesy Icarus). As in Figure 7, the dissipation is expressed as a function of the second-order Love number $k_{2}$, which evaluates the amplitude of the hydrostatic tidal deformation, and the tidal quality factor $Q$.

the hydrostatic pressure exerted by a non-dissipative homogeneous deep gaseous envelope boosts its amplitude in comparison to the case of a telluric core without any surrounding fluid region. However, the physical treatment of this solid dissipation he proposed was incomplete. Jean-Paul, Françoise, Valéry and myself were strongly motivated to consider this channel of dissipation as a serious candidate to explain the results of the astrometric observations because we demonstrated that the dissipation of the fluid equilibrium tide is not efficient enough to match the observed dissipation (by several orders of magnitude) while the dissipation of tidal (gravito-)inertial waves has an highly resonant behavior as demonstrated by Ogilvie \& Lin (2004) while the dissipation seems to have a smooth dependence on the tidal frequency for Encelade, Tethys, and Dione. In this framework, Remus et al. $(2012 \mathrm{~b}, 2015)$ were the first to provide coherence to the simplified bi-layer Dermott's model by using complex Love numbers and the correspondence principle (e.g. Tobie et al. 2005). They allow them to compute simultaneously the elastic deformation of the solid (see Fig. 10 - left panel) and the viscous dissipation in its bulk (see Fig. 10 - right panel). Using the simplest viscoelastic Maxwell rheology (see also the contribution by Gwanaël Boué), they were able to show that friction in the core can explain both the amplitude and the frequency-dependence of the strong dissipation observed in Jupiter and Saturn by Lainey et al. (2009, 2012). The main weakness of this work is our poor knowledge of the rheology of the core of giant planets in their extreme conditions of pressure and temperature. As explained in Remus et al. (2012b, 2015), this motivates the choice of the simplest 
Maxwell rheology and the exploration of plausible values of the core's rigidity and viscosity. Following their method, Storch \& Lai (2014) proposed this mechanism to explain the formation of hot Jupiters through circularization in a high-eccentricity migration scenario. Shoji \& Hussmann (2017) also demonstrated, taking into account the frequency-dependence of the core viscoelastic dissipation when solving the tidal orbital migration equations for the moons, that a strong tidal dissipation in Saturn does not imply necessarily young moons. Storch \& Lai (2015) also improved this bi-layer model by treating the case of a homogeneous core surrounded by a polytropic gas envelope. Lainey et al. (2017) achieved the more important generalization with taking into account density and compressibility radial profiles. The viscoelastic dissipation they computed is compatible with dissipation they measured at the tidal frequency of Enceladus, Tethys, and Dione, thanks to high precision astrometry as illustrated in Figure 10 (right panel). However, they also observed a strongest dissipation by one order of magnitude at the frequency of Rhea. The friction applied on the solid tide cannot explain this value if it is the source of dissipation for Enceladus, Tethys, and Dione. An additional dissipation mechanism operating in the fluid envelope should thus be invoked that points out the need to compute tidal dissipation in giant planets from their core to their surface.

Guenel et al. (2014) achieved a first step toward this direction. They used the simplified bi-layer model, where both the solid core and the fluid envelope are assumed to be homogeneous. Following the method by Ogilvie (2013), they computed the frequency-averaged viscoelastic tidal dissipation in the solid core and compared its amplitude to the frequency-averaged dissipation that Ogilvie computed for tidal inertial waves attractors propagating in spherical shells with an internal solid core. They demonstrated that these two mechanisms might lead to comparable tidal frictions when assuming plausible values for the rigidity of the core of giant planets. This shows how it is mandatory to compute tidal dissipation coherently in each solid, liquid, and gaseous planetary layer without any a priori. In this context, new improvements of the theory of the dissipation of tidal flows in the fluid regions of gaseous giant planets have been undertaken. Fuller et al. (2016); Luan et al. (2018) proposed that gravito-inertial waves propagating in a stably stratified ocean surrounding the core of giant planets can be trapped in resonances (Witte \& Savonije 2002). This mechanism can be a serious candidate to interpret the astrometric observations. It provides a natural explanation in the case of Saturn for the dissipation rates measured at the frequencies of Enceladus, Tethys, and Dione, but also for the dissipation at the frequency of Rhea, which cannot be explained by a solid friction mechanism. André et al. (2017), proposed that the presence of double-diffusive instabilities can also enhance the dissipation of tides when compared to the case of a "standard" fully convective envelope. The development of numerous stably-stratified layers due to stabilizing gradients of heavy elements (e.g. Leconte \& Chabrier 2012, and Fig. 11), allows the excitation, propagation, and dissipation of gravito-inertial waves in addition to inertial waves that can enhance the tidal dissipation. Finally, Mathis et al. 2016 proposed that rapid rotation may lead to a less efficient convective turbulent friction applied on tidal waves. Their resonances are then shaper with stronger amplitude 


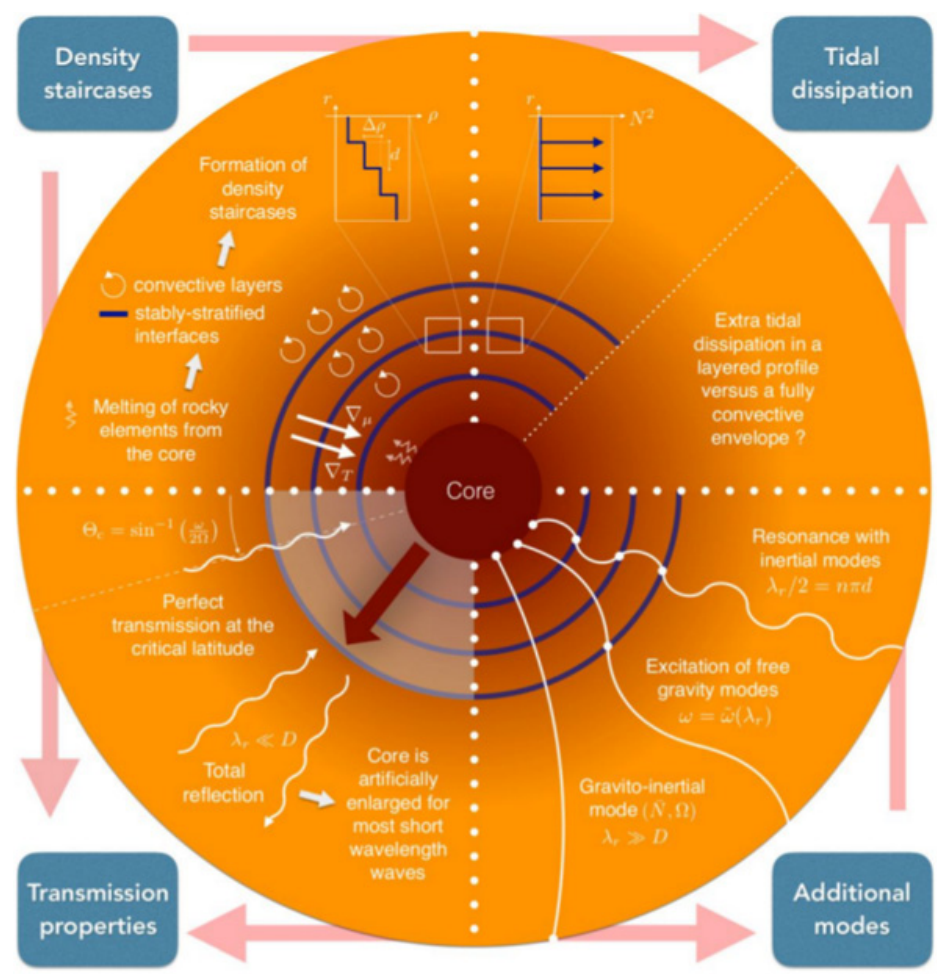

Fig. 11. Sketch presenting the complex situation of a gaseous giant planet in which double-diffusive instabilities take place. North-west quarter: how density staircases are possibly created. South-west quarter: main properties of the transmission of tidal internal gravito-inertial waves. South-east quarter: additional modes that could potentially be observed thanks to the seismology of planets. North-east quarter: the question of an enhanced tidal dissipation.

(Auclair Desrotour et al. 2015) that may explain rapid variations of the dissipation as a function of the tidal frequency as observed in the case of Rhea.

With the recent important progresses, which have been obtained thanks to JUNO for our understanding of the complex internal structure and dynamics of gaseous giant planets (Wahl et al. 2017; Guillot et al. 2018; Kaspi et al. 2018), we are only at the infancy of a coherent modeling of tidal dissipation in these objects. More should be done as in the case of stars to also take into account wave-turbulence nonlinear interactions, zonal flows, and magnetic fields.

\section{Boundary conditions}

As it has been shown previously, tidal interactions excite flows (i.e. the equilibrium tide, the dynamical tide, and fluid movements that result from their instabilities). 


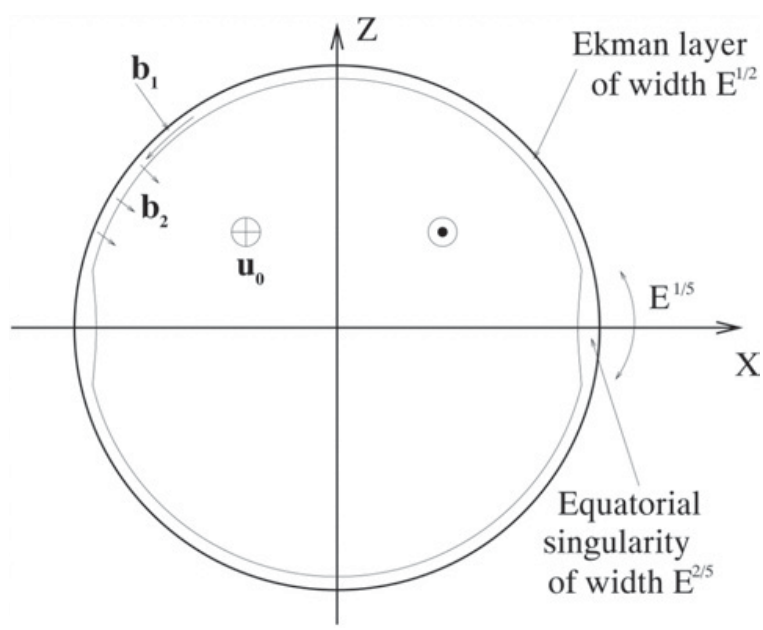

Fig. 12. Schematic view of Ekman boundary layers that can be important at boundaries of planetary cores but not at the free surfaces of stars and giant planets (taken from Rieutord \& Zahn (1997), in which details concerning boundary velocity fields, $\mathbf{b}_{1}$ and $\mathbf{b}_{2}$, are given; courtesy the Astrophysical Journal).

Then, depending on the internal structure of the studied body, boundary conditions, and particularly at the surface and near solid/fluid interfaces (in planetary interiors) should be examined carefully. Indeed, boundary layer strongly sheared flows can develop there, that may lead to a strong dissipation. Let us first consider the case of the free surface of stars or of planets. Tassoul \& Tassoul (1992a,b, 1997) have proposed that Ekman boundary layers take place and lead to an important viscous dissipation. However, Jean-Paul and Michel Rieutord pointed that such boundary is a free surface with stress-free boundary conditions for the velocity field and thus that the dissipation related to associated boundary layers will be very weak (Rieutord 1992; Rieutord \& Zahn 1997). However, if we consider the solid/fluid interfaces, we are in the case of no-slip boundary conditions that correspond to the classical Ekman boundary layers (see Fig. 12) where a strong viscous dissipation may occur, particularly if the studied region is turbulent. As a conclusion on boundary flows, one has thus to remember that solid/fluid regions (at the top of rocky/icy cores of giant planets and at boundaries of liquid cores in telluric planets) may host strong viscous dissipation while this is not the case below the free surface of stars or planets with a fluid envelope. At solid-fluid boundaries, we must also point that couplings between the fluid dynamical tide (inertial or gravito-inertial) and the (an)-elastic tide in solid regions must be studied in a near future since these may modify the related tidal dissipation.

\section{Conclusions}

As a conclusion, Jean-Paul Zahn was the founder of the modern theories for the equilibrium and the dynamical tides in stellar interiors. He developed their 
formalisms in his $\mathrm{PhD}$ (Zahn 1966a,b,c) for the equilibrium tide and during the seventies (Zahn 1970, 1975) for the dynamical tide in stably stratified radiation zones with important successes when their predictions (Zahn 1977, 1989) were confronted to observations of close binary early-type stars (North \& Zahn 2003), young late-type binaries (Zahn \& Bouchet 1989), and binary stars involving a giant component (Verbunt \& Phinney 1995; Beck et al. 2018). He was the first to propose a prescription for the loss of efficiency of the turbulent friction applied on the equilibrium tide by convective flows in the case of the "rapid" tides where the tidal period is short compared to the convective overturning time (Zahn 1966c, 1989). He also examined the link between tidal torques and the rotational and chemical evolution of binary stars (Zahn 1994; Cayrel de Strobel et al. 1994). Finally, he demonstrated with Michel Rieutord the inefficiency of tidal Ekman boundary layers at the free surface of stars and planets (Rieutord \& Zahn 1997).

These works, which were devoted to the study of close binary (and multiple) stars were then broadly used within the community studying exoplanetary systems. Indeed, many of them are compact (i.e. with short periods) with important tidal interactions that shape their orbital architecture while the rotational evolution of the host $\operatorname{star}(\mathrm{s})^{1}$ can be impacted by the presence of planetary companion(s). In some studies, Jean-Paul's prescriptions were directly applied to interpret the observations. But more importantly, his works constitute the root of a large number of important studies of the dynamical tide in (differentially) rotating stellar and planetary convective regions, which is constituted of tidal inertial waves, and of the dynamical tide in (differentially) rotating stably stratified stellar and planetary layers like stellar radiation zones, the atmospheres of giant and telluric planets, and the potentially deep oceans of telluric exoplanets (we refer the reader to the complete reviews by Ogilvie 2014; Le Bars et al. 2015; Mathis 2018, and references therein).

The last PhD thesis of Françoise Remus supervised by Jean-Paul has closed the loop of his seminal work on tides in stars and planets. First, we revisited his $\mathrm{PhD}$ work on the equilibrium tide in convective stellar (and planetary) regions (Remus et al. 2012b). We were also strongly involved in the Encelade international team, which has focused its activity on an interdisciplinary study of the system of Saturn, the favorite research area of one of Jean-Paul's best friend, Pr. André Brahic. In this team, we proposed a new model for the viscoelastic dissipation of solid tides in the dense central core of gaseous giant planets (Remus et al. 2012b, 2015). This has opened new lines of investigation of tidal dissipation mechanisms in these objects in our solar system and outside. They will benefit from each available progress on our knowledge of their internal structure and dynamics as for instance in the case of Jupiter with the JUNO space mission.

As a conclusion, Jean-Paul's work constitutes the pedestal of the ab-initio physical modeling of tidal dissipation in stars and more generally in celestial bodies hosting thick fluid regions. Each of us working in this research area has the honor to walk on the paths he has drawn.

\footnotetext{
1 Those can be binary or multiple stars.
} 


\section{Quelques derniers mots}

Cher Jean-Paul,

Par ce dernier hommage, c'est l'ensemble des étudiants et jeunes chercheurs qui ont eu la chance unique de collaborer avec toi qui te témoigne leur immense reconnaissance. Ainsi, depuis l'achèvement de ta thèse en 1966, tu as croisé puis partagé le chemin de nombre d'entre nous depuis la préparation de nos thèses et tout le long de nos carrières nous témoignant la même amitié fidèle, la même générosité, le même soutien indéfectible et attentif. Ainsi, nous pouvons témoigner que chaque jeune chercheur s'intéressant aux objets célestes en rotation depuis les étoiles, les planètes, les disques d'accrétion jusqu'aux expériences de laboratoire bénéficia toujours de ta part d'une immense bienveillance, d'une écoute attentive et d'une volonté puissante de transmettre avec pédagogie tes méthodes si originales et si fines pour répondre aux questions complexes que les astres nous soumettent chaque jour. C'est ainsi que tu t'intéressas aux travaux d'Arlette Rocca et de Michel Rieutord sur les interactions de marées dans les étoiles doubles. Puis, tu encadras avec ton ami fidèle Juri les travaux de Jean Latour, de Josep Massaguer et de Niel Hurlburt sur la convection turbulente. Tu dirigeas ensuite la thèse de Bérengère Dubrulle sur la turbulence dans les disques d'accrétion, sujet d'intérêt commun avec Suzy, dont la thèse de Denis Richard constitua la continuation et l'aboutissement. Le temps de la rotation et du mélange des étoiles vint avec ton soutien aux travaux de Corinne Charbonnel puis les thèses de Suzanne Talon et d'Ana Palacios. Simultanément, la compréhension de notre astre, le Soleil, émergea avec l'héliosismologie, et tu lançais Sacha Brun sur la compréhension magnétohydrodynamique de notre étoile et de ses analogues, sujet que tu partageas avec lui et ses étudiants dont Antoine Strugarek. Puis, ce fut mon tour, tout d'abord avec les marées, puis les échanges de moment cinétique dans les intérieurs stellaires, qui nous amenèrent à encadrer ensemble la thèse de Françoise Remus sur les marées tandis que tu étudiais l'aplatissement des étoiles avec Clément Ranc. L'ensemble de ces noms et de tous les étudiants et jeunes chercheurs que je n'ai pas nommés, qu'ils m'en excusent, auxquels tu témoignas ton soutien fort et indéfectible, témoigne ce que l'Astrophysique française et mondiale, et tout particulièrement les jeunes, te doivent. Tel un arbre puissant, tu lanças de nombreuses branches dont les contributions sont aujourd'hui reconnues. Ainsi, les étudiants de tes étudiants poursuivent des voies aussi riches que variées en utilisant les méthodes dites "à la Zahn", filles de l'Ecole que tu as formée et qui se transmettent et se transmettront de générations en générations.

Nous n'oublierons jamais tout ce que tu nous as appris et nous poursuivrons de manière volontaire ton oeuvre que tu as bâti brillamment en nous faisant bénéficier de tes exceptionnelles qualités humaines. Ainsi, en plus du formateur et de l'enseignant magnifique que tu étais, tu devins au fil du temps pour chacun d'entre nous un ami proche, un membre de la famille. Depuis les quais des gares d'Angleterre où tu portais les ours en peluche des enfants de Sacha jusqu'à cette église alsacienne où tu fus mon témoin de mariage, vous nous reçûmes toujours 
avec Suzy chez vous comme des membres de votre famille. Nous n'oublierons jamais ta gentillesse exceptionnelle, ta grande culture, ton amour pour la musique classique, l'Opéra et ta région natale l'Alsace, ainsi que la grande ouverture dont tu témoignas toujours lors de nos longues conversations sur de nombreux sujets que tu qualifias parfois avec espièglerie d'agréables récréations. Ton attention et ton affection à notre égard et pour nos proches contribuèrent de manière forte et inoubliable à notre épanouissement personnel et professionnel, ce dont nous te sommes infiniment reconnaissants.

A cette heure triste et douloureuse, je me souviens que nous n'avons jamais évoqué l'après de chacun d'entre nous, surement par pudeur ou par peur d'y penser. Ainsi, suivant les convictions de tout à chacun, nous te dirons adieu ou simplement au revoir mais dans tous les cas un immense merci répondant unanimement au titre d'un article dans L'Astronomie que tu écrivis en 1964 intitulé "Appel aux jeunes".

Au revoir Jean-Paul. Tu entres aujourd'hui dans le Panthéon des hommes et des femmes qui ont façonné ce que je suis et chaque jour à venir sera rempli du merveilleux souvenir des moments partagés à tes côtés, de tout ce que tu nous as donné, et de ta voix si douce à nos coeurs.

Hoenheim, Terre d'Alsace, le 29/07/2015.

S. Mathis acknowledges funding by the European Research Council through ERC grant SPIRE 647383. This work was also supported by the Programme National de Planétologie (CNRS/INSU) and the PLATO CNES grant at Département d'Astrophysique (CEASaclay).

\section{References}

Albrecht, S., Winn, J.N., Johnson, J.A., et al., 2012, ApJ, 757, 18

Alterman, Z., Jarosch, H., \& Pekeris, C.L., 1959, Proc. R. Soc. London Ser. A, 252, 80

André, Q., Barker, A.J., \& Mathis, S., 2017, A\&A, 605, A117

Arras, P., \& Socrates, A., 2010, ApJ, 714, 1

Astoul, A., Mathis, S., Baruteau, C., et al., 2018, in SF2A-2018: Proceedings of the Annual meeting of the French Society of Astronomy and Astrophysics, ed. P. Di Matteo, F. Billebaud, F. Herpin, N. Lagarde, J.-B. Marquette, A. Robin, \& O. Venot, in press

Auclair-Desrotour, P., Laskar, J., \& Mathis, S., 2017, A\&A, 603, A107

Auclair-Desrotour, P., Le Poncin-Lafitte, C., \& Mathis, S., 2014, A\&A, 561, L7

Auclair-Desrotour, P., \& Leconte, J., 2018, A\&A, 613, A45

Auclair-Desrotour, P., Mathis, S., Laskar, J., \& Leconte, J., 2018, A\&A, 615, A23

Auclair Desrotour, P., Mathis, S., \& Le Poncin-Lafitte, C., 2015, A\&A, 581, A118

Baraffe, I., 2005, Space Sci. Rev., 116, 67

Baraffe, I., Chabrier, G., Fortney, J., \& Sotin, C., 2014, Protostars and Planets VI, 763

Barker, A.J., 2011, MNRAS, 414, 1365

Barker, A.J., 2016, MNRAS, 459, 939 
Barker, A.J., Dempsey, A.M., \& Lithwick, Y., 2014, ApJ, 791, 13

Barker, A.J., \& Lithwick, Y., 2013, MNRAS, 435, 3614

Barker, A.J., \& Lithwick, Y., 2014, MNRAS, 437, 305

Barker, A.J., \& Ogilvie, G.I., 2009, MNRAS, 395, 2268

Barker, A.J., \& Ogilvie, G.I., 2010, MNRAS, 404, 1849

Baruteau, C., \& Rieutord, M., 2013, J. Fluid Mech., 719, 47

Batygin, K., 2012, Nature, 491, 418

Beck, P.G., Mathis, S., Gallet, F., et al., 2018, MNRAS, 479, L123

Benbakoura, M., Réville, V., Brun, A.S., Le Poncin-Lafitte, C., \& Mathis, S., 2019, A\&A, 621 , in press

Benz, W., Ehrenreich, D., \& Isaak, K., 2017, CHEOPS: CHaracterizing ExOPlanets Satellite (Springer), 84

Bolmont, E., Gallet, F., Mathis, S., et al., 2017, A\&A, 604, A113

Bolmont, E., \& Mathis, S., 2016, Celest. Mech. Dyn. Astron., 126, 275

Bolmont, E., Raymond, S.N., Leconte, J., \& Matt, S.P., 2012, A\&A, 544, A124

Bolton, S.J., Adriani, A., Adumitroaie, V., et al., 2017, Science, 356, 821

Braviner, H.J., \& Ogilvie, G.I., 2014, MNRAS, 441, 2321

Braviner, H.J., \& Ogilvie, G.I., 2015, MNRAS, 447, 1141

Brun, A.S., García, R.A., Houdek, G., Nandy, D., \& Pinsonneault, M., 2015, Space Sci. Rev., 196, 303

Bryan, G.H., 1889, Philos. Trans. Royal Soc. London Ser. A, 180, 187

Cayrel de Strobel, G., Cayrel, R., Friel, E., Zahn, J.-P., \& Bentolila, C., 1994, A\&A, 291, 505

Cébron, D., \& Hollerbach, R., 2014, ApJ, 789, L25

Chabrier, G., \& Baraffe, I., 2007, ApJ, 661, L81

Charnoz, S., Crida, A., Castillo-Rogez, J.C., et al., 2011, Icarus, 216, 535

Chernov, S.V., Papaloizou, J.C.B., \& Ivanov, P.B., 2013, MNRAS, 434, 1079

Claret, A., \& Cunha, N.C.S., 1997, A\&A, 318, 187

Damiani, C., \& Mathis, S., 2018, A\&A, 618, A90

Dermott, S.F., 1979, Icarus, 37, 310

Duquennoy, A., \& Mayor, M., 1991, in Lecture Notes in Physics, Berlin Springer Verlag, Vol. 390, Bioastronomy: The Search for Extraterrestial Life - The Exploration Broadens, ed. J. Heidmann \& M.J. Klein, 39

Duquennoy, A., Mayor, M., \& Mermilliod, J.-C., 1992, Binaries as Tracers of Star Formation, ed. A. Duquennoy \& M. Mayor, 52

Efroimsky, M., \& Lainey, V., 2007, J. Geophys. Res. (Planets), 112, E12003

Favier, B., Barker, A.J., Baruteau, C., \& Ogilvie, G.I., 2014, MNRAS, 439, 845

Fielding, D.B., McKee, C.F., Socrates, A., Cunningham, A.J., \& Klein, R.I., 2015, MNRAS, 450, 3306

Fuller, J., Luan, J., \& Quataert, E., 2016, MNRAS, 458, 3867

Gallet, F., Bolmont, E., Bouvier, J., Mathis, S., \& Charbonnel, C., 2018, A\&A, 619, A80

Gallet, F., Charbonnel, C., Amard, L., et al., 2017, A\&A, 597, A14

Gillon, M., Triaud, A.H.M.J., Demory, B.-O., et al., 2017, Nature, 542, 456 
Giuricin, G., Mardirossian, F., \& Mezzetti, M., 1984, A\&A, 131, 152

Goldreich, P., \& Keeley, D.A., 1977, ApJ, 211, 934

Goldreich, P., \& Nicholson, P.D., 1989, ApJ, 342, 1079

Goldreich, P., \& Soter, S., 1966, Icarus, 5, 375

Goodman, J., \& Dickson, E.S., 1998, ApJ, 507, 938

Goodman, J., \& Lackner, C., 2009, ApJ, 696, 2054

Greenberg, R., 2009, ApJ, 698, L42

Gu, P.-G., \& Ogilvie, G.I., 2009, MNRAS, 395, 422

Guenel, M., Baruteau, C., Mathis, S., \& Rieutord, M., 2016a, A\&A, 589, A22

Guenel, M., Mathis, S., Baruteau, C., \& Rieutord, M., 2016b, in Cambridge Workshop on Cool Stars, Stellar Systems, and the Sun, Vol. 19, Proceedings of the 19th Cambridge Workshop on Cool Stars, Stellar Systems, and the Sun, ed. G.A. Feiden, 1-4

Guenel, M., Mathis, S., \& Remus, F., 2014, A\&A, 566, L9

Guillot, T., 1999, Science, 296, 72

Guillot, T., Lin, D.N.C., Morel, P., Havel, M., \& Parmentier, V., 2014, in EAS Publications Series, Vol. 65, EAS Publications Series, 327

Guillot, T., Miguel, Y., Militzer, B., et al., 2018, Nature, 555, 227

Hansen, B.M.S., 2012, ApJ, 757, 6

Husnoo, N., Pont, F., Mazeh, T., et al., 2012, MNRAS, 422, 3151

Hut, P., 1980, A\&A, 92, 167

Ivanov, P.B., Papaloizou, J.C.B., \& Chernov, S.V., 2013, MNRAS, 432, 2339

Jackson, B., Greenberg, R., \& Barnes, R., 2008, ApJ, 678, 1396

Kaspi, Y., Galanti, E., Hubbard, W.B., et al., 2018, Nature, 555, 223

Kaula, W.M., 1962, AJ, 67, 300

Kerswell, R.R., 2002, Ann. Rev. Fluid Mech., 34, 83

Khaliullin, K.F., \& Khaliullina, A.I., 2010, MNRAS, 401, 257

Koch, R.H., \& Hrivnak, B.J., 1981, AJ, 86, 438

Lagage, P.-O., 2015, European Planetary Science Congress 2015, held 27 September - 2 October, 2015 in Nantes, France, Online at http://meetingorganizer. copernicus .org/EPSC2015, id.EPSC2015-757, 10, EPSC2015

Lai, D., 2012, MNRAS, 423, 486

Lai, D., Foucart, F., \& Lin, D.N.C., 2011, MNRAS, 412, 2790

Lainey, V., Arlot, J.-E., Karatekin, Ö., \& van Hoolst, T., 2009, Nature, 459, 957

Lainey, V., Jacobson, R.A., Tajeddine, R., et al., 2017, Icarus, 281, 286

Lainey, V., Karatekin, Ö., Desmars, J., et al., 2012, ApJ, 752, 14

Latham, D.W., Stefanik, R.P., Torres, G., et al., 2002, AJ, 124, 1144

Le Bars, M., Cébron, D., \& Le Gal, P., 2015, Ann. Rev. Fluid Mech., 47, 163

Leconte, J., \& Chabrier, G., 2012, A\&A, 540, A20

Leconte, J., \& Chabrier, G., 2013, Nat. Geosci., 6, 347

Li, G., \& Winn, J.N., 2016, ApJ, 818, 5

Lin, Y., \& Ogilvie, G.I., 2018, MNRAS, 474, 1644

Love, A.E.H., 1911, Some Problems of Geodynamics (Cambridge University Press)

Luan, J., Fuller, J., \& Quataert, E., 2018, MNRAS, 473, 5002 
MacDonald, G.J.F., 1964, Rev. Geophys. Space Phys., 2, 467

Mathieu, R.D., \& Mazeh, T., 1988, ApJ, 326, 256

Mathieu, R.D., Meibom, S., \& Dolan, C.J., 2004, ApJ, 602, L121

Mathis, S., 2009, A\&A, 506, 811

Mathis, S., 2013, in Lecture Notes in Physics, Berlin Springer Verlag, Vol. 865, Lecture Notes in Physics, Berlin Springer Verlag, ed. M. Goupil, K. Belkacem, C. Neiner, F. Lignières, \& J.J. Green, 23

Mathis, S., 2015a, in SF2A-2015: Proceedings of the Annual meeting of the French Society of Astronomy and Astrophysics, ed. F. Martins, S. Boissier, V. Buat, L. Cambrésy, \& P. Petit, 401

Mathis, S., 2015b, A\&A, 580, L3

Mathis, S., 2018, Tidal Star-Planet Interactions: A Stellar and Planetary Perspective (Springer), 24

Mathis, S., 2016, Auclair-Desrotour, P., Guenel, M., Gallet, F., \& Le Poncin-Lafitte, C., A\&A, 592, A33

Mathis, S., \& Le Poncin-Lafitte, C., 2009, A\&A, 497, 889

Mathis, S., Le Poncin-Lafitte, C., \& Remus, F., 2013, in Lecture Notes in Physics, Berlin Springer Verlag, Vol. 861, Lecture Notes in Physics, Berlin Springer Verlag, ed. J. Souchay, S. Mathis, \& T. Tokieda, 255

Mayor, M., \& Queloz, D., 1995, Nature, 378, 355

Mazeh, T., Perets, H.B., McQuillan, A., \& Goldstein, E.S., 2015, ApJ, 801, 3

Mazevet, S., Tsuchiya, T., Taniuchi, T., Benuzzi-Mounaix, A., \& Guyot, F., 2015, Phys. Rev. B, 92, 014105

McQuillan, A., Mazeh, T., \& Aigrain, S., 2013, ApJ, 775, L11

Meibom, S., \& Mathieu, R.D., 2005, ApJ, 620, 970

Meibom, S., Mathieu, R.D., \& Stassun, K.G., 2006, ApJ, 653, 621

Mermilliod, J.-C., Rosvick, J.M., Duquennoy, A., \& Mayor, M., 1992, A\&A, 265, 513

Mirouh, G.M., Baruteau, C., Rieutord, M., \& Ballot, J., 2016, J. Fluid Mech., 800, 213

Moutou, C., Boisse, I., Hébrard, G., et al., 2015, in SF2A-2015: Proceedings of the Annual meeting of the French Society of Astronomy and Astrophysics, ed. F. Martins, S. Boissier, V. Buat, L. Cambrésy, \& P. Petit, 205-212

Murray, C.D., \& Dermott, S.F., 1999, Solar system dynamics, Cambridge University Press (Cambridge University Press)

Nettelmann, N., Fortney, J.J., Moore, K., \& Mankovich, C., 2015, MNRAS, 447, 3422

North, P., \& Zahn, J.-P., 2003, A\&A, 405, 677

Ogilvie, G.I., 2005, J. Fluid Mech., 543, 19

Ogilvie, G.I., 2013, MNRAS, 429, 613

Ogilvie, G.I., 2014, ARA\&A, 52, 171

Ogilvie, G.I., \& Lesur, G., 2012, MNRAS, 422, 1975

Ogilvie, G.I., \& Lin, D.N.C., 2004, ApJ, 610, 477

Ogilvie, G.I., \& Lin, D.N.C., 2007, ApJ, 661, 1180

Papaloizou, J.C.B., \& Savonije, G.J., 1997, MNRAS, 291, 651

Penev, K., Sasselov, D., Robinson, F., \& Demarque, P., 2007, ApJ, 655, 1166

Pollack, J.B., Hubickyj, O., Bodenheimer, P., et al., 1996, Icarus, 124, 62 
Polycarpe, W., Saillenfest, M., Lainey, V., et al., 2018, A\&A, 619, A133

Press, W.H., 1981, ApJ, 245, 286

Rauer, H., Catala, C., Aerts, C., et al., 2014, Exper. Astron., 38, 249

Remus, F., Mathis, S., \& Zahn, J.-P., 2012a, A\&A, 544, A132

Remus, F., Mathis, S., Zahn, J.-P., \& Lainey, V., 2012b, A\&A, 541, A165

Remus, F., Mathis, S., Zahn, J.-P., \& Lainey, V., 2015, A\&A, 573, A23

Ricker, G.R., Winn, J.N., Vanderspek, R., et al., 2015, J. Astron. Telescopes, Instrum. Syst., 1, 014003

Rieutord, M., 1992, A\&A, 259, 581

Rieutord, M., 2015, Fluid Dynamics: An Introduction, Springer-Verlag (Springer)

Rieutord, M., \& Valdettaro, L., 1997, J. Fluid Mech., 341, 77

Rieutord, M., \& Zahn, J.-P., 1997, ApJ, 474, 760

Rocca, A., 1987, A\&A, 175, 81

Rocca, A., 1989, A\&A, 213, 114

Rogers, T.M., \& Lin, D.N.C., 2013, ApJ, 769, L10

Salpeter, E.E., 1973, ApJ, 181, L83

Sana, H., \& Evans, C.J., 2011, in IAU Symposium, Vol. 272, Active OB Stars: Structure, Evolution, Mass Loss, and Critical Limits, ed. C. Neiner, G. Wade, G. Meynet, \& G. Peters, 474-485

Savonije, G.J., \& Witte, M.G., 2002, A\&A, 386, 211

Shoji, D., \& Hussmann, H., 2017, A\&A, 599, L10

Sinclair, A.T., 1983, in Astrophysics and Space Science Library, Vol. 106, IAU Colloq. 74: Dynamical Trapping and Evolution in the Solar System, ed. V.V. Markellos \& Y. Kozai, 19-25

Stevenson, D.J., 1975, Phys. Rev. B, 12, 3999

Stevenson, D.J., 1979, Geophys. Astrophys. Fluid Dyn., 12, 139

Stevenson, D.J., 1985, Icarus, 62, 4

Storch, N.I., \& Lai, D., 2014, MNRAS, 438, 1526

Storch, N.I., \& Lai, D., 2015, MNRAS, 450, 3952

Strugarek, A., Bolmont, E., Mathis, S., et al., 2017, ApJ, 847, L16

Tassoul, J.-L., \& Tassoul, M., 1992a, ApJ, 395, 259

Tassoul, M., \& Tassoul, J.-L., 1992b, ApJ, 395, 604

Tassoul, M., \& Tassoul, J.-L., 1997, ApJ, 481, 363

Teitler, S., \& Königl, A., 2014, ApJ, 786, 139

Terquem, C., Papaloizou, J.C.B., Nelson, R.P., \& Lin, D.N.C., 1998, ApJ, 502, 788

Tinetti, G., Drossart, P., Eccleston, P., et al., 2018, Exper. Astron., 46, 135

Tobie, G., Grasset, O., Lunine, J.I., Mocquet, A., \& Sotin, C., 2005, Icarus, 175, 496

Verbunt, F., \& Phinney, E.S., 1995, A\&A, 296, 709

Wahl, S.M., Hubbard, W.B., Militzer, B., et al., 2017, Geophys. Res. Lett., 44, 4649

Wei, X., 2016, ApJ, 828, 30

Wei, X., 2018, ApJ, 854, 34

Wilson, H.F., \& Militzer, B., 2012, Phys. Rev. Lett., 108, 111101

Winn, J.N., Fabrycky, D., Albrecht, S., \& Johnson, J.A., 2010, ApJ, 718, L145 
Witte, M.G., \& Savonije, G.J., 1999a, A\&A, 341, 842

Witte, M.G., \& Savonije, G.J., 1999b, A\&A, 350, 129

Witte, M.G., \& Savonije, G.J., 2002, A\&A, 386, 222

Wu, Y., 2005a, ApJ, 635, 674

Wu, Y., 2005b, ApJ, 635, 688

Zahn, J.P., 1966a, Annales d'Astrophysique, 29, 313

Zahn, J.P., 1966b, Annales d'Astrophysique, 29, 313

Zahn, J.P., 1966c, Annales d'Astrophysique, 29, 489

Zahn, J.P., 1970, A\&A, 4, 452

Zahn, J.-P., 1975, A\&A, 41, 329

Zahn, J.-P., 1977, A\&A, 57, 383

Zahn, J.-P., 1989, A\&A, 220, 112

Zahn, J.-P., 1994, A\&A, 288, 829

Zahn, J.-P., 2013, in Lecture Notes in Physics, Berlin Springer Verlag, Vol. 861, Lecture Notes in Physics, Berlin Springer Verlag, ed. J. Souchay, S. Mathis, \& T. Tokieda, 301

Zahn, J.-P., \& Bouchet, L., 1989, A\&A, 223, 112 